\title{
Eva BerTRAND
}

Sciences Po-CERI, Paris

\section{Quand l'humanitaire entre en politique : l'exemple du ministère russe des Situations d'Urgence}

Les deux crises majeures que sont la catastrophe nucléaire de Tchernobyl, le 26 avril 1986, et le tremblement de terre qui touche la ville arménienne de Spitak, le 7 décembre 1988, marquent l'entrée de la Russie dans la "société du risque ». Le risque n'est plus seulement perçu comme un danger venant de l'extérieur, mais peut être généré de l'intérieur par la société qu'il menace (Beck 2008 : 399). Comme le rappellent les tremblements de terre qui ont détruit les villes d'Achkhabad en 1948, et de Tachkent en 1966, la coulée de boue qui s'est abattue sur Alma-Ata en 1973, ou encore le tsunami qui a touché la côte orientale russe en 1952, ces risques ne sont, bien sûr, pas nouveaux en soi. Le changement qui s'opère au milieu des années 1980 est le fruit de la « confluence historique » entre deux événements désastreux en termes humains, politiques et économiques, et un contexte politique d'ouverture (Profiriev, 1998 : XIII).

L'entrée en politique du problème des situations d'urgence s'inscrit, en effet, dans le contexte de la glasnost qui met en partie fin au mutisme d'État autour de ces événements. Ce contexte est aussi celui d'une décomposition du contrôle de l'information qui ouvre la voie à une couverture médiatique des catastrophes, ainsi que des accidents du quotidien. Tchernobyl illustre bien ce tournant : la catastrophe ne peut rester secrète. Dès les premiers jours, des reporters se sont rendus sur place 
et des informations sont très vite diffusées dans les médias (Mandrillon 1991 : 1384). Après Tchernobyl, mais aussi après Spitak, les faiblesses du système soviétique sont mises au jour aussi bien à l'intérieur qu'à l'extérieur du pays, qu'il s'agisse de l'incroyable état d'impréparation des sauveteurs, de l'incapacité à venir en aide aux victimes, ou de l'extraordinaire lenteur des canaux officiels d'information (Elie 2013 : 220-223). Outre la vulnérabilité de la population, c'est la force de délégitimation que représentent les catastrophes pour le pouvoir en place qui est révélée par ces deux événements.

L'essor politique de la notion de situation d'urgence se traduit par la création d'un ministère spécialement en charge de la gestion des catastrophes. Créé en 1994, le ministère des Situations d'Urgence (MTchS) se définit comme l'organe fédéral du pouvoir exécutif de la Fédération de Russie habilité à décider des questions de défense de la population et du territoire face aux situations d'urgence d'origine naturelle ou technologique, à l'intérieur comme à l'extérieur de l'État russe.

Cette structure originale est restée dans l'angle mort des recherches sur la Russie et des disaster studies ${ }^{1}$. Les principaux apports sont ceux des historiens de l'environnement qui comme Marie-Hélène Mandrillon, et plus récemment Marc Elie et Laurent Coumel, se sont intéressés au rapport des autorités soviétiques à l'environnement, à la protection de la nature et à la gestion des catastrophes. Le MTchS en tant que structure de force est également entré dans le champ des chercheurs s'intéressant aux questions de défense et de gestion des conflits, comme Bettina Renz et Aude Merlin.

Nous avons, quant à nous, fait le choix d'appréhender le MTchS dans le cadre d'une approche de politique publique, entendue comme l'" appréhension au concret de l'État en action » (Hassenteufel 2008 : 7). Il s'agit de saisir la genèse de la politique de l'urgence russe et la mise en œuvre des dispositifs institutionnels qui l'incarnent ${ }^{2}$. À l'exemple des travaux de Vincent Dubois sur le ministère français de la Culture (Dubois 1999), ou ceux de Pierre Lascoumes sur le ministère français de l'Environnement (Lascoumes 1999), l'objectif est de comprendre comment s'opère

1 Un ouvrage en langue anglaise doit toutefois être ici mentionné : Boris Porfiriev, Disaster Policy and emergency management in Russia. New York : Nova Science Publishers Inc., 1998. Et plus récemment : Boris Porfiriev, Greg Simons, Crises in Russia. Contemporary Management Policy and Practice from a Historical Perspective, Burlington : Ashgate, 2012.

2 Nous nous rapprochons ainsi des travaux de Milanna Streltsina, qui a soutenu en 2004 à l'Université Européenne de Saint-Pétersbourg une thèse intitulée Institucional'nye uslovija sozdanija pravitel'stvennyh vedomstv v Rossii $i$ SŠA : sravnitel'nyj analiz (Conditions institutionnelles de création des structures gouvernementales en Russie et aux États-Unis : étude comparative) analysant parallèlement le MTchS et son homologue américain, l'Agence Fédérale de gestion des urgences (FEMA). 
l'institutionnalisation d'une nouvelle fonction d'État justifiée par la nécessité concrète de protéger une population, mais aussi par des enjeux régaliens, voire bureaucratiques, de stabilité des institutions dans un contexte de réformes. Suivant une logique chronologique, nous reviendrons sur les antécédents soviétiques du MTchS, pour interroger ensuite sa création, son extension, ainsi que les logiques structurelles et personnelles qui ont favorisé son inscription dans le tissu institutionnel postsoviétique sur le temps long.

L'accès et la rareté des sources ont conditionné le choix de nous placer du côté de la création institutionnelle, plutôt que d'opter pour une approche plus sociologique. Le MTchS se caractérisant par des logiques de fonctionnement quasi-militaires, dans un contexte où la culture du secret est très forte, il ne nous a pas été possible de nous reposer uniquement sur un travail d'investigation direct. La dizaine d'interviews que nous avons pu réaliser avec des représentants du ministère, notamment au niveau régional, a été complétée par le dépouillement des publications officielles, en particulier les revues Graždanskaja zaščita et Spasatel', et le site Internet de l'institution (<http:// www.mchs.gov.ru>). Si elles ne dévoilent pas les tensions internes au ministère, ces sources écrites permettent néanmoins une approche en terme d'histoire et d'évolution institutionnelle, et offrent un premier accès à une structure en apparence hermétique.

Après avoir examiné ses antécédents et son développement institutionnel, nous nous pencherons sur le ministère « en action » du début des années 1990 à nos jours, en montrant que, paradoxalement, la popularité de la structure tient moins à ses activités en temps de guerre et de catastrophe qu'à sa capacité à communiquer autour de ses fonctions, de ses valeurs, mais aussi de la personnalité d'un homme, l'ancien ministre des Situations d'Urgence, Sergueï Choïgou.

\section{Le sauveteur à épaulettes : institutionnalisation du ministère russe des Situations d'Urgence}

\section{Les racines soviétiques de l'humanitaire d'État russe}

À l'origine de la notion de « situation d'urgence », qui apparaît avec le MTchS et les structures qui l'ont directement précédé, se trouve celle de « défense civile ». Les deux guerres mondiales et, en particulier, les bombardements américains de Nagasaki et Hiroshima, ont mis au jour la nécessité de développer des institutions en charge de la protection de la population civile. Ce besoin s'est incarné successivement dans deux structures dont le MTchS est en partie l'héritier : le Système d'État de défense antiaérienne locale (Obščesojuznaja sistema mestnoj protivovozdušnoj oborony SSSR), créé en 1932, devenu en 1961 celui de la Défense civile (Graždanskaja oborona) rattaché au ministère de la Défense. Si ces premières organisations visent à 
répondre aux risques d'attaques nucléaires, chimiques et bactériologiques extérieures, les catastrophes majeures des années 1980 témoignent de l'existence d'un autre type de menace : des événements soudains, sans ennemis, produits de l'intérieur, à faible probabilité, et occasionnant des pertes conséquentes à la fois humaines, matérielles et financières.

En juillet 1989, pour répondre aux défis que pose la gestion de ces catastrophes naturelles et technologiques, un Comité d'État aux Situations d'Urgence ou GKTchS ${ }^{3}$ est institué au niveau du gouvernement central de l'Union Soviétique. Ce Comité, instauré à la toute fin de l'ère soviétique, apparaît comme la matrice du futur MTchS de Russie, avec lequel il partage nombre de caractéristiques, en particulier une fonctionnalité orientée vers les différentes facettes de la gestion des catastrophes, et une grande proximité du pouvoir présidentiel (Elie 2013 : 235-236).

Des institutions de gestion des catastrophes se structurent parallèlement au niveau des républiques, comme le Corps russe de sauveteurs (Rossijskij korpus spasatelej) créé fin 1990. Le 30 juillet 1991, ce Corps de sauveteurs est transformé en Comité d'État de la RSFSR à la Défense Civile, aux Situations d'Urgence et à la Liquidation des Conséquences des Sinistres ${ }^{4}$, placé sous l'autorité directe du Président de la RSFSR. Suite au décret présidentiel no 221 du 19 novembre 1991, le Comité d'État aux Situations d'Urgence et le personnel de la Défense civile de la RSFSR, dépendant du ministère de la Défense, fusionnent. Le GKTchS de la RSFSR est alors chargé d'intégrer le personnel de la Défense civile de la RSFSR, et en raison du démantèlement de l'Union en décembre 1991, il reprend également les structures du GKTchS de l'URSS (locaux, personnels - Elie 2013 : 236).

L'évolution institutionnelle des organes de gestion de l'urgence en URSS, et en Russie soviétique, semble suggérer le passage d'une structure de défense orientée vers des menaces militaires et nucléaires, à des structures qui restent statutairement militaires, mais développent des activités de sauvetage en situation de crises civiles. Nous sommes donc, même si cela n'est pas nommé comme tel, face une politique humanitaire. L'objectif final est bien de « préserver la vie dans le respect de la dignité, [et de] restaurer l'homme dans ses capacités de choix » (Brauman 1995 : 9). C'est ainsi sur la base des articles 20, 35, 41 et 42 de la Constitution de 1993 qui affirment le droit des citoyens russes à la vie, à la santé et à la propriété, que le 10 janvier 1994, le GKTchS devient le ministère de la Fédération de Russie de la Défense Civile, des

3 En russe : Gosudarstvennyj komitet po delam graždanskoj oborony, črezvyčajnym situacijam, i likvidacii posledstvij stihijnyh bedstvij.

4 En russe : Gosudarstvennyj komitet RSFSR po delam graždanskoj oborony, črezvyčajnym situacijam, i likvidacii posledstvij stihijnyh bedstvij. 
Situations d'Urgence et de la Liquidation des Conséquences des Sinistres ${ }^{5}$ (Porfiriev $1998: 103)$.

Les fonctions du MTchS, parfois qualifié de "ministère des Catastrophes ", procèdent de la notion de «situation d'urgence ». Ce terme recouvre les situations qui, résultant d'« un accident, d'une catastrophe naturelle ou d'autres désastres susceptibles d'endommager, ou ayant endommagé des infrastructures, peuvent être la source de troubles pour la santé humaine ou l'environnement, ainsi que de pertes matérielles considérables, et marquent une rupture dans les conditions de vie normales d'une population $»^{6}$.

Le ministère est en charge d'une politique humanitaire, mais comme tend à le rappeler sa proximité du pouvoir présidentiel précédemment évoquée, d'une politique humanitaire d'État, c'est-à-dire partagée entre des missions de sauvetage et une allégeance forte au pouvoir en place. Pour l'État, l'humanitaire ne peut se concevoir hors du politique (Dubuet 2003 : 56), et le rapport à la personne n'est pas primordial. Comme le formule le vocabulaire officiel, la mission essentielle de cette institution est la « liquidation des conséquences des catastrophes » (likvidacija posledstvij stihijnyh bedstvij). Dans la droite ligne de l'idéologie soviétique, il s'agit de démontrer la capacité de l'homme à maîtriser et dominer la nature (Merlin 2003 : 88). L'enjeu, par-delà le sauvetage des victimes, est d'effacer toute trace de non maîtrise face à l'imprévu. L'idéologie marxiste-léniniste du rapport entre l'homme et la nature, et du rôle de la technique dans ce rapport, informe les institutions en charge des catastrophes et de l'urgence (Merlin 2003 : 88). Cette conception et ce vocabulaire sont une constante dans la politique de l'urgence, qu'il s'agisse de l'URSS ou de la Russie postsoviétique.

\section{Création d'un ministère de l'urgence entre civils et militaires}

Outre les événements catastrophiques mentionnés plus haut, la création d'un ministère en charge des catastrophes s'ancre aussi dans les logiques de réorganisation institutionnelle du nouvel État russe (Merlin 2003 : 87). Suite à la Constitution du 12 décembre 1993, qui précise que seuls les ministres fédéraux peuvent prendre part au gouvernement, le nombre de ministères et de comités d'État est considérablement réduit (Lesage 1993 : 619). Parallèlement, à l'initiative de Boris Eltsine, une partie des comités d'État est transformée en ministères. C'est le cas du GKTchS qui devient donc MTchS. Sa création intervient dans un contexte où le nombre des « structures de

5 En russe : Ministerstvo Rossijskoj Federacii po delam graždanskoj oborony, črezvyčajnym situacijam, i likvidacii posledstvij stihijnyh bedstvij.

6 Acte fédéral « Sur la protection de la population et des territoires contre les urgences naturelles et technologiques » de 1994. 
force » (silovye struktury ${ }^{7}$ ) s'est considérablement accru. Au nombre de trois sous le régime soviétique - ministère de l'Intérieur, ministère de la Défense et Comité pour la Sécurité d'État (KGB) -, ces structures passent au nombre de quatorze lors des réformes des années $1990^{8}$, pour ensuite se stabiliser à huit structures permanentes en 2006 (cf. tableau 1). La réforme des institutions de sécurité a notamment pour but de fragmenter et de décentraliser les organes existants et ainsi de réduire leur influence (Volkov $2002: 84$ ).

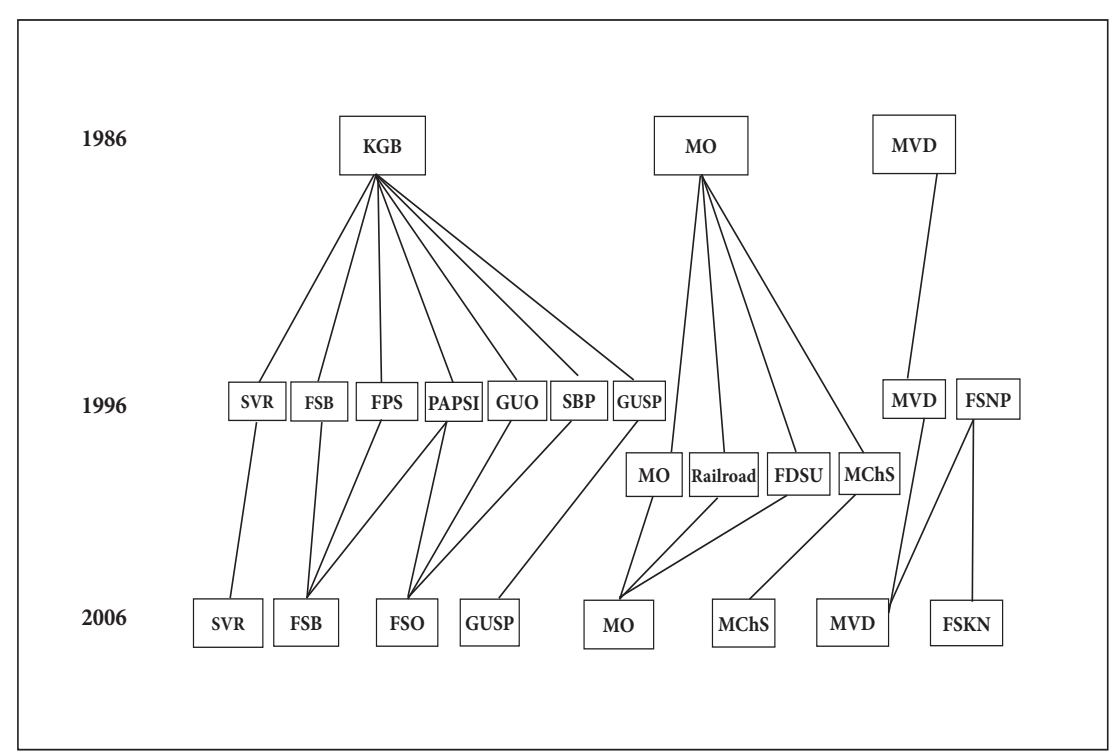

Tableau 1 : Réforme des structures de forces (Taylor 2007 : 2)

Dans un contexte où la détention de ressources administratives est gage de pouvoir, le découpage ministériel est l'objet d'un affrontement direct entre détenteurs

7 Il s'agit des ministères et structures d'État qui, en raison de leur capacité militaire, ou, tout au moins, de leur proximité avec les intérêts premiers de l'État, sont placés directement sous la tutelle du Président de la Fédération de Russie.

8 Service de renseignements extérieurs (SVR), Service de sécurité fédérale (FSB), Service fédéral des frontières (FPS), Service fédéral d'information et de communication (FAPSI), Directorat principal des gardes (GUO), Service de sécurité du Président (SBP), Directorat principal des programmes spéciaux du Président (GUSP), ministère de la Défense (MO), Service des chemins de fer, Administration fédérale de construction des routes (FDSU), ministère des Situations d'Urgence (MTchS/MChS), ministère de l'Intérieur (MVD), Service fédéral de la police des impôts (FSNP) et ministère de la Justice (non mentionné dans le schéma ci-après). 
et convoiteurs de ces ressources. Le morcellement des ministères est perçu comme l'occasion d'obtenir une part des rentes bureaucratiques (Favarel-Garrigues et Startsev 2007 : 71). Dans cette lutte, les fondateurs du MTchS9 se révèlent capables de profiter de la fenêtre d'opportunité ouverte par les réformes et d'acquérir le statut de ministère.

Le MTchS semble, de fait, disposer d'une série d'avantages comparatifs au regard des autres structures de force. Si une certaine légitimité semble accordée à la structure et à ses promoteurs, elle bénéficie également largement des carences des autres institutions. Les principaux ministères soviétiques (Intérieur, Défense et KGB) ont, en effet, tous trois été impliqués dans la tentative de putsch orchestrée contre le pouvoir en août 1991 (Taylor 2007 : 1). Le MTchS bénéficie également de l'obsolescence, de la désorganisation et du déficit d'image qui frappent les ex-structures de force soviétiques. Ces carences ne permettaient pas d'envisager de les doter de nouvelles fonctions en matière de gestion des catastrophes naturelles et technologiques.

La création du MTchS participe aussi d'une logique qui vise à affaiblir plus spécifiquement le ministère de la Défense (Tsyganok 2010), miné par des affaires de corruption et d'illégitimité ${ }^{10}$. Néanmoins, cette redistribution des fonctions de sécurité ne s'est pas déroulée sans heurts. L'idée, par exemple, de doter le MTchS d'unités chargées des opérations de maintien de la paix, prérogative du ministère de la Défense, a fait l'objet d'une vive opposition de la part de Pavel Gratchev, ministre de la Défense d'alors, qui a conduit à l'abandon du projet (Thomas 1995 : 232). L'extension des fonctions et des troupes du MTchS s'est donc opérée au gré des rapports de force entretenus avec les autres ministères.

Lors de sa création, le MTchS compte un personnel de 70000 hommes, dont 23000 proviennent des troupes de Défense civile du ministère de la Défense - ces troupes ont été placées sous l'autorité du GKTchS dès décembre 1991. À la différence des contingents militaires usuels, ces unités sont destinées aux opérations de protection de la population en cas d'urgence, d'accident ou de catastrophe naturelle.

9 Un petit groupe d'acteurs dirigeants et influents s'est attelé à défendre un nouveau modèle de sécurité d'État. Ce noyau regroupe plusieurs leaders actuels ou passés du ministère. Il s'agit de Iouri Vorobiev, Premier vice-ministre du MTchS jusqu'en 2007 ; Mikhaïl Faleev, vice-ministre du MTchS de 1996 à 2006 ; Iouri Brajnikov vice-ministre du MTchS jusqu'en 2004 ; Ruslan Tsalikov, Premier vice-ministre du MTchS de 2007 à 2012 ; Andreï Legochin, directeur adjoint du département des forces anti-incendie du MTchS ; Vladimir Legochin, ancien directeur adjoint de la section de sauveteurs d'élite Tsentrospas ; et bien sûr Sergueï Choïgou ministre de la Défense depuis 2012, ministre du MTchS de 1994 à 2012 et fondateur du Corps russe de sauveteurs dès 1990.

10 Le Général Pavel Gratchev, ministre de la Défense de 1992 à 1996, avait ainsi été surnommé 'Pacha Mercedes' en raison des trafics de matériel militaire dans lesquels il s'était trouvé impliqué. 
Le transfert de ces troupes était motivé par la volonté de réduire leur non-emploi au sein de leur institution d'origine (Renz 2007a : 149). Les hommes ont conservé leur grade et années de service. Parmi le personnel du MTchS, $40 \%$ des hommes sont des militaires, parmi lesquels $40 \%$ sont des soldats contractuels. Le MTchS se présente ainsi comme « une organisation militaire au profil humanitaire » (Renz 2007a : 151).

Les éléments civils sont toutefois dominants au sein du ministère par leur nombre, et par le choix qui a été fait d'un ministre civil, que ce soit Sergueï Choïgou, ministre de 1994 à 2012, ou Vladimir Pouchkov, ministre depuis 2012. L'hybridité de l'institution est néanmoins source de tensions, les différents statuts engendrant des traitements administratifs distincts et une éthique ou, tout au moins, un esprit différent. En février 2006, Sergueï Choïgou avait annoncé le lancement d'une série de réformes visant à transformer d'ici 2011 les éléments militaires du ministère en troupes civiles de maintien de l'ordre, sans leur attribuer de rôle en temps de guerre (Renz 2007a : 151). Mais devenu ministre de la Défense en 2012, il a au contraire évoqué la possibilité de faire passer le MTchS sous la tutelle du ministère de la Défense (Muhin 2013). La tension entre dimension civile et militaire est donc une constante dans le fonctionnement du ministère. Elle n'est cependant pas propre à la Russie : en France par exemple, l'histoire du corps des sapeurs-pompiers est jalonnée de tensions entre civil et militaire, mais aussi entre troupes armées et désarmées (Rohart 2005).

\section{Champs de compétences et extension des ressources administratives}

Si les initiateurs d'une structure ministérielle dédiée à la gestion des situations d'urgence semblent avoir bénéficié de ce qui peut être décrit comme un effet d'aubaine bureaucratique, résultat des réformes de la décennie 1990, l'extension ultérieure du MTchS invalide le caractère strictement conjoncturel de cette tendance. Le ministère a progressivement acquis de nouveaux secteurs de compétence. Depuis sa création, son appareil bureaucratique s'est doté de départements supplémentaires, de domaines de compétences additionnels et d'une administration élargie au niveau régional, même si la réforme administrative de 2004 a conduit à une réduction de son appareil administratif fédéral (cf. tableau 2). 
Eva BERTRAND

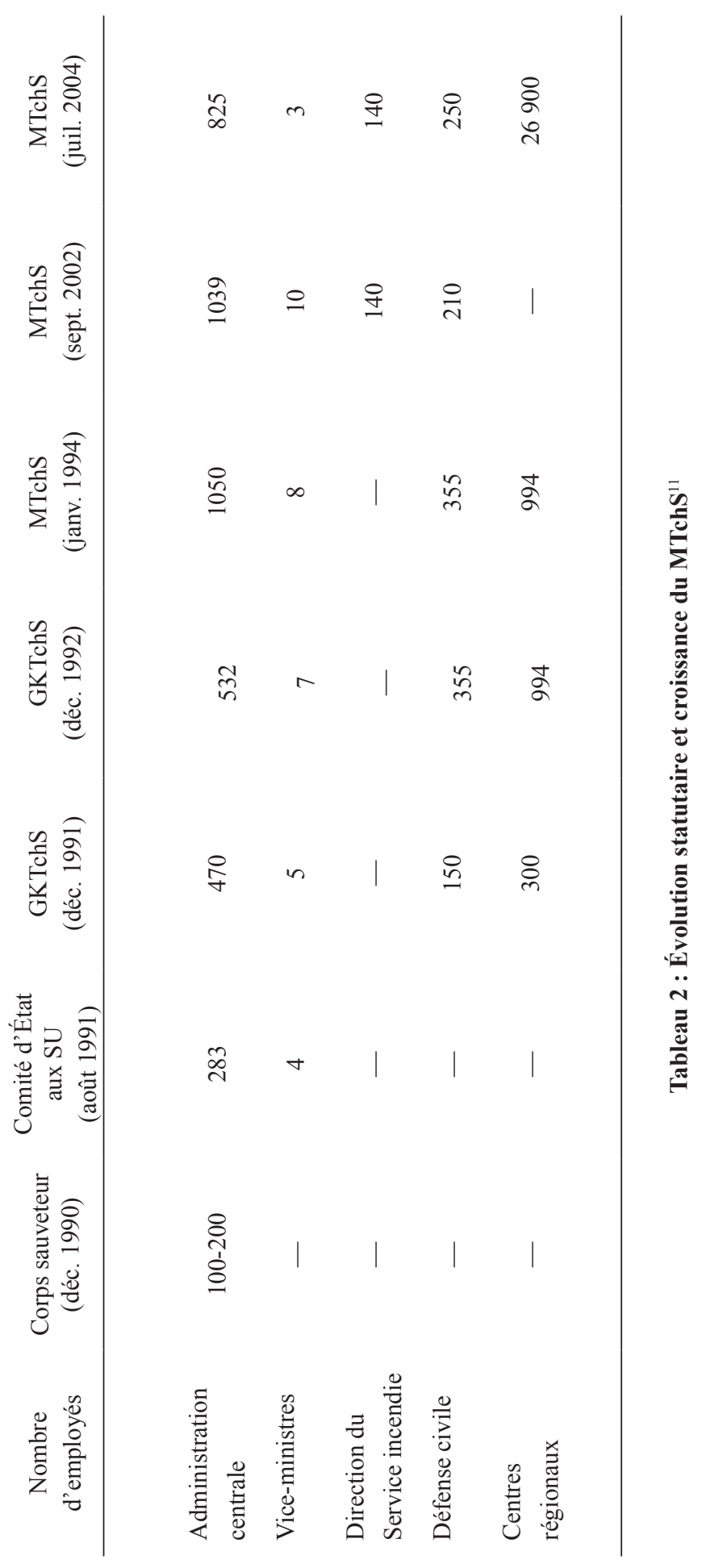

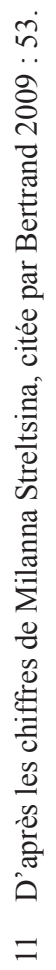


Les fonctions de base du MTchS correspondent aux missions classiquement confiées aux troupes de défense civile : prévention et gestion des catastrophes naturelles et technologiques. Ces fonctions premières se sont étoffées à la fois en termes de supports techniques, d'équipement et de personnel.

L'accent a été mis sur le développement des établissements médicaux, des organismes de prévention et de prévision des risques naturels et technologiques. En matière de santé, par exemple, à la fin des années 1990, le MTchS a hérité du Centre russe de médecine écologique (Vserossijskij centr ekologičeskoj mediciny - VTsEM), initialement placé sous la tutelle du ministère de la Santé pour prendre en charge les victimes de Tchernobyl. En 1999, le VTsEM s'est étoffé d'un Centre d'aide psychologique d'urgence (Centr Ekstrennoj Psihologičeskoj pomošči) en charge de l'accompagnement psychologique des sauveteurs et des victimes des catastrophes durant lesquelles le MTchS est mobilisé ${ }^{12}$.

Outre le domaine de la santé, le MTchS s'est doté, en 2006, d'un centre de prévention des risques : le Centre national de contrôle des situations de crise (Nacional'nyj centr upravlenija $v$ krizisnyh situacijah) en charge du contrôle des opérations de prévention et de liquidation des situations d'urgence, ainsi que $\mathrm{du}$ travail de veille et d'information relatif aux risques. En matière de formation, un Centre spécifique de préparation des sauveteurs du MTchS (Rossijskij Centr podgotovki spasatelej) est créé le 7 mai 1996. Ce centre, qui devient en 1998 le Quarantième centre de préparation des sauveteurs du MTchS (40-j Rossijskij Centr podgotovki spasatelej), vise à former les sauveteurs du ministère à toutes les étapes de leur carrière. Le MTchS de Russie acquiert ainsi un poids institutionnel croissant, reposant sur la création et l'incorporation de structures périphériques. Le petit Corps russe de Sauveteurs des années 1990 s'est ainsi mué en l'une des principales structures de force du pays, comme le souligne Sergueï Choïgou :

Personne ne pensait que la création du Corps de sauveteurs dans les années à venir prendrait l'allure du service d'urgence russe... Aujourd'hui le MTchS est une structure flexible et ramifiée, présente dans la quasi-totalité du pays. C'est un dispositif de 70 000 spécialistes, plus de 120 équipes de sauveteurs-secouristes - une dans chaque région, mais deux dans certaines régions plus difficiles. En outre, le MTchS c'est une aviation, une médecine, et encore beaucoup d'autres choses (Šojgu $2000: 5$ ).

Au début des années 2000, le poids institutionnel du MTchS s'est encore renforcé suite à deux importants changements. Tout d'abord, le décret présidentiel $\mathrm{n}^{\circ} 1309$ du 9 novembre 2001 décide de transférer au MTchS les 220000 hommes du Service d'État de lutte anti-incendie, auparavant rattachés au ministère de l'Intérieur. Le poids administratif du MTchS s'est alors trouvé quasiment multiplié par trois. De par

12 En 2004, ce centre est devenu indépendant. Arrêté ministériel du MTchS du 25 avril $2003, n^{\circ} 218$ « Sur la création d'un service psychologique du MTchS de Russie ». 
son nombre d'hommes, le MTchS est devenu la quatrième structure de force du pays après le ministère de la Défense, le ministère de l'Intérieur et le Service Fédéral de Sécurité, ancien KGB (Taylor 2007 : 2).

Ce développement s'est accompagné d'une extension des fonctions du ministère. Il s'est vu confier de nouvelles missions de prévention et de sauvetage, ainsi qu'un rôle d'inspection et de contrôle relatif à la sécurité incendie. L'attribution au MTchS de l'Inspection d'État des bateaux de faible tonnage ${ }^{13}$ tend également à modifier le rôle du ministère, en lui conférant des missions de sécurité, de prévention des accidents et des travaux de sauvetage en mer, mais aussi de classification et d'enregistrement des navires, de formation et d'attribution des permis de navigation, de régulation des parcs à bateaux, de contrôles écologiques et de sécurité des plages. L'extension des fonctions du MTchS s'est donc accompagnée d'une redéfinition de son rôle vers des fonctions plus préventives et sécuritaires, et s'est faite autour d'une appréhension large de la notion de situation d'urgence. Ces nouvelles fonctions recouvrent également des domaines (inspection de sécurité, attribution de permis) traditionnellement sources d'une importante corruption. Outre le poids institutionnel du ministère, c'est son champ de captation des « ressources administratives » directement transformables en ressources financières qui semble s'être élargi au fil des réformes.

\section{Le ministère en action : entre logiques institutionnelles, personnalisation et communication}

\subsection{Sauveteurs ou soldats : quel rôle en temps de guerre?}

Si comme nous l'avons précédemment mentionné, les missions de maintien de la paix ne font pas partie des attributions du MTchS, le ministère s'est néanmoins vu conférer un rôle en temps de guerre en matière de premiers secours, d'assistance médicale et d'évacuation des civils ${ }^{14}$. Ces fonctions sont un héritage du GKTchS dont la création, à la toute fin des années 1980, est concomitante de l'éclosion de multiples conflits ethniques sur le sol de l'Union soviétique. Il est ainsi intervenu pour la première fois durant l'été 1992 lors des affrontements opposant Géorgiens, Ossètes et Abkhazes. Le GKTchS apparaît alors, selon Sergueï Choïgou, comme la seule structure gouvernementale apte à assurer cette mission :

13 Oukase présidentiel $n^{\circ} 991$ du 28 août 2003 et résolution gouvernementale $n^{\circ} 47$ du 6 février 2004. 
Dès le début, il a été décidé que le GKTchS ne devait pas être impliqué en politique. Cependant, quand les conflits ethniques ont éclaté sur les frontières et sur le territoire russes, et que nous pouvions voir de nos yeux des milliers de victimes, une question s'est posée : qui va les aider ? Si ce n'est pas le GKTchS, qui le fera ? (...) C'est ainsi que nous avons reçu la mission d'aider les populations dans les zones de conflits ethniques... Le travail du GKTchS en zone de conflit incluait l'évacuation des personnes blessées, leur installation dans des zones sécurisées, ainsi que l'apport d'une aide médicale et humanitaire. C'est ainsi que cela s'est passé dans presque toutes les régions qui ont souffert des conséquences des conflits ethniques : l'Ossétie du Nord et du Sud, l'Abkhazie, l'Ingouchie et les autres. (Streltsina $2006: 12$ )

Le MTchS hérite de cette fonction d'aide humanitaire en temps de conflit. Il est intervenu durant les deux conflits tchétchènes et en Ossétie du Sud en août 2008, et intervient actuellement dans l'Est de l'Ukraine.

En 1994-1996, puis en 1999-2000, l'intervention du MTchS en Tchétchénie comprenait différents volets : hébergement, apport de nourriture, rétablissement des systèmes d'eau et d'électricité, assistance médicale, mais aussi opérations de déminage et coopération avec les agences des Nations Unies et la Croix Rouge (Renz 2007a :149). Il est cependant difficile d'apprécier l'ampleur réelle de l'aide apportée par le ministère des Situations d'Urgence en Tchétchénie. La lecture des actions entreprises par les différents organes d'État russes y est particulièrement ardue dans la mesure où main droite (bras armé) et main gauche (assistance, protection) de l'État agissent de concert sur un même territoire (Merlin 2003 : 95-98).

Toutefois, le rôle affiché du MTchS ainsi que l'uniforme et le non-armement de ses troupes visent à rendre nettement visible la différence entre sauveteur et soldat. Voici comment un représentant du Comité International de la Croix Rouge le décrit à partir de son expérience durant le conflit osséto-géorgien de 2008 :

Visiblement ce sont des gens qui s'affichent non armés, qui s'affichent civils, qui s'affichent pompier international [...] et c'est extrêmement subtil, beaucoup plus subtil qu'un militaire français qui va avec son fusil distribuer du riz. [...] Les Russes avec le système du MTchS séparent clairement les tâches : il y a celui qui s'occupe du maintien de la paix, des tâches militaires, et celui qui est en charge de la sécurité civile, et de l'assistance aux populations civiles. C'est bien joué en termes d'organisation ${ }^{15}$.

Lors du conflit qui a opposé la Géorgie à l'Ossétie du Sud en août 2008, le MTchS de Russie a apporté une assistance humanitaire à la population ossète. Afin de venir en aide aux quelques 34000 réfugiés ossètes, le $\mathrm{MTchS}$ a ouvert 61 points de prise en charge, organisé l'approvisionnement en nourriture, et le rétablissement des systèmes 
de distribution d'eau, de gaz et d'électricité, ainsi que des opérations de déminage dans la région en guerre ${ }^{16}$. Le délégué de la Croix-Rouge continue :

Pendant le conflit ossète, en août 2008, le MTchS de Russie a déployé un contingent complet pour ce qui est assistance et infrastructure, que ce soit pour l'assainissement de l'eau, l'aide médicale, la nourriture, la distribution de vêtements, toute la panoplie de ce que l'on appelle l'assistance. [...] En termes d'assistance, le MTchS est une organisation extrêmement bien organisée, quasiment paramilitaire. Ils avaient des hélicoptères, des camions, ils avaient cet espèce d'uniforme de pompier bleu marine, qui fait qu'à la fin du mois d'aout, Tskhinvali était une ville bleue. Il y a même des journalistes, je crois un journaliste de Libération, qui évoquait la ville comme « schtroumpfland». Ils étaient partout : dans les hôpitaux, dans les magasins, dans la réfection des tuyaux, des canaux, ils réinstallaient l'électricité ${ }^{17}$.

De par ses modalités d'action et la palette de ses activités, le MTchS tend à s'approprier et à monopoliser le champ humanitaire, rendant accessoire toute action non-gouvernementale. Durant le conflit de l'été 2008, par exemple, le MTchS, présent en Ossétie du Sud, a interdit l'accès de la région aux organisations non gouvernementales internationales, qui sont restées cantonnées à Vladikavkaz, en Ossétie du Nord. Seuls deux acteurs humanitaires étaient présents en Ossétie du Sud : le MTchS et la Croix Rouge ${ }^{18}$. Un représentant de Médecins sans frontières souligne les difficultés auxquelles s'est heurtée son équipe en voulant agir en Ossétie du Sud :

Pendant la rencontre, la réponse est claire : « Nous n'avons pas besoin de votre aide » [...] pour le gouvernement russe, elles [les ONG] sont le signe de sa propre incompétence à régler ses problèmes intérieurs, un signe de ses propres carences, donc une forme d'hostilité naturelle naît à l'égard des acteurs humanitaires non étatiques ${ }^{19}$.

Face à cette éviction des organisations non gouvernementales, les activités humanitaires du MTchS semblent constituer un vecteur d'affirmation de la puissance régalienne de l'État dans ses frontières, mais aussi au-delà.

En effet, le MTchS participe à l'intégration des structures de sécurité russes dans un environnement mondial post Guerre froide. La mise en œuvre d'un humanitaire d'État civil apparaît comme l'un des marqueurs de l'ouverture de la Russie à l'international. Immédiatement après sa création, le MTchS a participé à des missions humanitaires et de coopération à l'étranger. Pour des raisons politiques, ces missions pouvaient difficilement être confiées au ministère de la Défense (Streltsina 2006 : 14). Dès 1994,

16 Interview de Sergueï Chapochnikov, directeur du département de protection civile du MTchS, $1^{\text {er }}$ octobre 2008, Ria Novosti.

Entretien réalisé par l'auteur le 17 mars 2009.

18 Selon les représentants du Comité International de la Croix Rouge interviewés par l'auteur le 17 et le 19 mars 2009. 
les hommes du MTchS apportent une aide humanitaire au Rwanda (distribution de nourriture, restauration des infrastructures), puis en 1999, une opération en partenariat avec la Suisse, la Grèce et l'Autriche est menée dans les Balkans (distribution de nourriture, de médicaments et autres produits de première nécessité, ouverture d'un hôpital à Prokuplje en Serbie pour apporter une aide médicale aux réfugiés, et opérations de déminage). Une opération de grande ampleur a été conduite par le MTchS en Afghanistan, en 2001-2002, avec notamment la création d'une base humanitaire dotée d'un hôpital à Kaboul.

Outre ces missions d'apport d'aide humanitaire, les troupes du MTchS interviennent dans l'évacuation des ressortissants russes se trouvant en zone de conflit : comme en 1994 au Yémen, au Liban en 2006, ou plus récemment en Syrie en 2013. Concernant ce dernier conflit, il est intéressant de noter que par-delà le seul rapatriement des binationaux, le MTchS a été impliqué dans l'apport de matériel en Syrie. Un partenariat a également été établi avec la Croix Rouge Internationale pour la mise en place d'un programme humanitaire local financé par la Russie ${ }^{20}$. Il s'agit d'une première dans la coopération institutionnalisée entre la Croix Rouge Internationale et le MTchS. Ces quelques exemples permettent de mettre en lumière deux aspects centraux du travail du MTchS à l'international. D'une part, les missions entreprises à l'étranger par le ministère permettent à la Russie de sortir de sa sphère classique d'influence, de l' « étranger proche ». D'autre part, elles se présentent comme un outil de présence stratégique dans des zones géopolitiquement clés. Le ministère apparaît donc comme le bras non armé de la politique étrangère russe, mettant en œuvre une politique humanitaire engagée en zone de conflit ou post-conflit.

\subsection{Un minist(e) re comme les autres?}

La décennie 1990 a vu éclore différentes structures de force à la pérennité toute relative. Le MTchS est l'une des rares institutions à avoir subsisté. Ce succès peut tout d'abord être expliqué par la forte proximité existant entre le ministère et le pouvoir présidentiel russe, mais aussi par la personnalité du ministre, qui a créé et véritablement incarné cette structure jusqu'en 2012. Le MTchS est un ministère qui occupe une place à part dans le gouvernement russe. En tant que « ministère de force », il fait partie de l'exécutif et est placé sous la tutelle directe du Président de la Fédération. Mais cette singularité est accentuée par le fait que son ministre, en sa qualité de membre non permanent du Conseil de Sécurité de l'Administration présidentielle, est directement soumis au Président (Porfiriev 1998 : 113). L'urgence apparaît ainsi comme un domaine réservé, au cœur du pouvoir politique russe.

20 Entretien réalisé par l'auteur le 3 décembre 2013 avec un représentant du Comité International de la Croix Rouge à Moscou. 
Cette place à part se retrouve dans la confiance que la population accorde au ministre en charge des Situations d'Urgence. Le cas de Sergueï Choïgou, parfois qualifié de ministre le plus longtemps en poste (Taylor 2007 : 4), est à ce titre particulièrement intéressant. Sergueï Choïgou n'est pas seulement un entrepreneur bureaucratique à succès, il est également un ministre apprécié par la population, et occupe souvent l'une des trois premières places dans les classements portant sur les personnalités préférées des Russes. Sa cote de popularité a, tout au long de son mandat au ministère des Situations d'Urgence, été l'une des plus élevées du gouvernement. Lors de la première campagne présidentielle de Vladimir Poutine, en 1999-2000, il a été choisi comme porte-parole du Parti Unité. Le choix du ministre des Situations d'Urgence comme représentant du nouveau parti du pouvoir n'est pas le fruit du hasard. Ce dernier est, comme le désigne la presse française au moment de la campagne, un « homme du clan » (L’humanité, 14 décembre 1999), engagé en politique dès l'époque soviétique. Cet engagement est, en partie, un héritage familial, son père ayant occupé le poste de vice-président du Conseil des ministres de la République de Touva (Ržeševskij 2005 : 10), d'où Sergueï Choigou est originaire. Lui-même est, dès 1988, deuxième secrétaire du Comité du Parti communiste de la ville d'Abakan. En 1989, il occupe le poste d'inspecteur du PCUS de la région de Krasnoïarsk. En 1990, il devient viceprésident du Comité d'État de la RSFSR pour l'architecture et la construction. Dans un contexte où les liens personnels et la loyauté apparaissent comme « des facteurs prédominants dans la nomination à des postes politiques » (Renz 2007b : 3), Sergueï Choïgou se présente comme un membre à part entière du système politique soviétique. En 1991, il est placé à la tête du Corps russe de sauveteurs, puis en novembre de la même année, Boris Eltsine le nomme Président du Comité d'État aux situations d'urgence, tout juste créé. Le 20 janvier 1994, il devient tout naturellement ministre des Situations d'Urgence.

Si institutionnellement le MTchS est proche du pouvoir suprême, Sergueï Choïgou est un proche des différents présidents qui se sont succédé depuis la chute de l'URSS, qu'il s'agisse de Boris Eltsine, dont il encadre la campagne présidentielle dans les régions en 1996, ou de Vladimir Poutine, dont il a soutenu la première élection. Ce soutien est du reste salué par le nouveau Président qui le nomme, en janvier 2000, vice-premier ministre, tout en lui laissant conserver son poste au MTchS. Sergueï Choïgou réaffirme, quant à lui, sa loyauté au pouvoir au cours de la seconde guerre de Tchétchénie. En février 2000, il est responsable de l'assistance fédérale apportée à la République. Durant cette mission, il soutient l'armée et la stabilité étatique, et défend la politique du gouvernement, en déclarant notamment que « critiquer les actions des troupes fédérales revient à trahir les intérêts du pays et de l'armée russe » (Karasik 2000 : 179). Ses positions patriotiques et son engagement au sein du parti Russie 
Unie $^{21}$ assurent son ancrage au cœur du pouvoir et confirment sa stature nationale, et par là même la stabilité de son ministère. Selon Milanna Streltsina :

Il est devenu un symbole du nouveau pouvoir présidentiel, et de la puissance du président. Choïgou est l'un des principaux piliers de ce pouvoir. Puis il a pris une position clé au sein du parti Russie Unie et a constitué un soutien de poids pour le régime du président, donc il est tout naturel que son ministère se développe ${ }^{22}$.

Cette autorité politique se double, comme nous l'avons évoqué plus haut, d'une forte popularité. Selon un sondage effectué durant la première campagne présidentielle de Vladimir Poutine, dans lequel les personnes interrogées devaient placer plusieurs figures publiques sur une échelle de 0 à 10 (de la plus forte désapprobation au plus fort assentiment), Sergueï Choïgou est le seul homme politique à obtenir plus de la moyenne. Ce sondage demandait également d'attribuer quatre traits de caractère aux personnalités évaluées. Le ministre des Situations d'Urgence s'est vu prêter les quatre qualités suivantes : intelligence, force, honnêteté et empathie avec les gens simples (Colton et McFaul 2000 : 218-219). Dans sa mise en scène médiatique usuelle, le ministre se présente ainsi en homme de terrain et d'action, éloigné des enceintes du pouvoir et proche du peuple. Il est présent sur tous les points chauds et n'hésite pas à jouer de cette présence dans la presse, ou devant les caméras de télévision. Cette exposition en homme de terrain s'accompagne du refus d'être défini comme un homme politique classique ou un technocrate. Il a d'ailleurs à plusieurs reprises décliné certaines fonctions politiques, comme le siège de député qui lui a été proposé en 1999 et en $2003^{23}$.

Tout au long des années 2000, la cote du ministre est restée stable. En 2007, par exemple, le centre de sondages VTsIOM a publié la cote de popularité des hommes politiques les plus appréciés de la Fédération. Dans ce classement, Sergueï Choïgou est en troisième position, juste après Vladimir Poutine et Dmitri Medvedev. En 2013, alors qu'il est devenu ministre de la Défense, succédant au très controversé Anatoli Serdioukov, Sergueï Choïgou est le ministre en qui les Russes ont le plus confiance (VTsIOM 2013). Si la popularité du ministère tient ainsi à un homme, elle semble toutefois aussi attachée à sa fonction. En effet, Vladimir Pouchkov, ministre des Situations d'Urgence depuis 2012, est en 2013 le troisième ministre en qui les Russes ont le plus confiance (VTsIOM 2013). Homme d'appareil, plutôt méconnu du grand public jusqu'à sa prise de fonction, Vladimir Pouchkov semble bénéficier de l'image positive du MTchS auprès de la population russe.

21 Dont il est co-président en 2001 et dont il devient en 2003 président du Conseil suprême. Entretien réalisé par l'auteur le 6 avril 2009.

23 Biographie de Sergueï Choïgou, http://lenta.ru/lib/14159636/ [consultée en novembre 2014]. 


\subsection{Une success story postsoviétique?}

2.4 La popularité du MTchS est ainsi sensiblement plus élevée que celle des autres structures de force. Ses hommes sont réputés pour leurs compétences, leur professionnalisme et leur sens des responsabilités (FOM 2002). De même, selon un sondage réalisé par le centre de recherche indépendant ROMIR dans la première moitié des années 2000 , à la question « quel ministère vous paraît travailler le plus efficacement ? », 25,8\% des personnes interrogées ont répondu en faveur du ministère des Situations d'Urgence contre 14,7 \% pour le ministère des Affaires Etrangères, $5,7 \%$ pour le ministère de l'Intérieur et 5,6\% pour le ministère de la Défense (Streltsina 2003 : 155). Néanmoins, cette image repose principalement sur une représentation construite par les médias. En effet, si $66 \%$ des personnes interrogées évaluent positivement le travail de ce ministère, $96 \%$ déclarent ne jamais avoir été en contact direct avec ses hommes (FOM 2002). Face à l'expérience concrète des catastrophes telles que les vastes incendies de l'été 2010, les inondations du Kuban en juillet 2012 ou encore de la région de l'Amour à l'automne 2013, les troupes du ministère se sont révélées insuffisamment équipées au niveau local, et la gestion de ces situations d'urgence a reposé essentiellement sur le ministère de la Défense et non sur le MTchS (Muhin 2013), contre lequel de nombreuses critiques se sont élevées.

Néanmoins, par-delà la capacité du MTchS à communiquer efficacement sur ses activités, son crédit auprès de la population peut être attribué à son inscription réussie dans la société et à sa faculté de se différencier des autres ministères de force. Cette différenciation résulte, en premier lieu, de la définition d'une fonction propre explicitée dans la devise du ministère : "Prévention, sauvetage et aide » (Predupreždenie, spasenie i pomošč). Ces fonctions sont associées à une profession, créée par les structures dont le MTchS est l'héritier : celle de sauveteur. Le sauveteur est perçu comme une figure symbolique valorisante, caractérisée par son dévouement, et son désintéressement. Cette dernière qualité semble particulièrement importante. Un officier du MTchS décrit le travail des sauveteurs comme une "vocation» :

Ils ne gagnent pas beaucoup d'argent. Ils ont tous un petit travail à côtée ${ }^{4}$.

Le désintéressement du sauveteur constitue l'un des topoï de l'imaginaire collectif, proche en ce sens de la «figure idéalisée et héroïsée du sapeur-pompier 'bénévole' » français (Rohart 2005). Dans l'imaginaire russe, cette figure se pose en rupture avec les représentants classiques des autres ministères de force, comme le militaire contractuel attaché à sa solde ou le milicien-policier extorqueur de « vziatki » (potsde-vin).

24 Entretien réalisé par l'auteur le 4 avril 2009. 
Par-delà les valeurs, l'identité du MTchS s'est forgée au travers d'éléments concrets de reconnaissance ou d'artefacts symboliques. Le 25 décembre 199725, un drapeau et un système héraldique spécifique au MTchS sont adoptés. De même, depuis 1995, le 27 décembre est le Jour des Sauveteurs de Russie ${ }^{26}$ - le 4 décembre restant le Jour de la Défense Civile. Cette date correspond à la création du premier Corps russe de Sauveteurs, le 27 décembre 1990. Outre cette fête annuelle, des manifestations plus ponctuelles sont organisées, comme par exemple la célébration des quinze ans du ministère, la nomination du sauveteur de l'année ou encore l'élection annuelle de Miss MTchS - qui, outre sa cocasserie, permet de souligner la place des femmes dans une institution majoritairement masculine.

Le sauveteur du MTchS serait ainsi l'équivalent du soldat soviétique pendant la Seconde Guerre mondiale : une représentation du héros de notre temps. Cela semble assez bien illustré dans le dernier film du réalisateur russe, Fiodor Bondartchouk, Stalingrad, sorti en Russie en octobre 2013. Ce film réalisé en l'honneur des soixantedix ans de la bataille de Stalingrad s'ouvre sur une intervention des troupes du MTchS au Japon, suite à un tremblement de terre. Un avion du MTchS atterrit et les hommes du ministère, dotés d'équipements ultramodernes, se lancent dans le sauvetage des sinistrés. Un lien direct est alors établi entre les soldats soviétiques qui se sont battus à Stalingrad et les sauveteurs du MTchS - le représentant du MTchS que l'on suit au début du film étant le fils d'un soldat mort à Stalingrad. Les soldats, qui hier ont sauvé la Patrie de la terreur nazie, se retrouvent aujourd'hui dans les sauveteurs intervenant face aux catastrophes naturelles.

\section{Conclusion}

Le MTchS apparaît ainsi comme l'un des quelques succès institutionnels des années 1990. Succès dont les facteurs sont multiples : avantages comparatifs, réseaux, personnalités influentes, ancrage social réussi et aptitude à communiquer. La bonne fortune du MTchS dépasse d'ailleurs les frontières russes, ses troupes intervenant dans la gestion des catastrophes naturelles et technologiques à l'étranger. Si la création du MTchS participe d'une volonté d'assurer l'État dans ses fonctions régaliennes en période de troubles institutionnels et politiques, l'attribution à ce ministère de

25 Conformément à l'oukase présidentiel, du 15 novembre 1997 n 1231 « Sur le drapeau et la signification héraldique - l'emblème du ministère de la Fédération de Russie pour la Défense civile, les situations d'urgence et la liquidation des conséquences des sinistres ».

26 Oukase présidentiel n 1306, du 25 novembre 1995, « Sur l'établissement du jour des sauveteurs de la Fédération de Russie ». 
fonctions internationales en fait l'instrument d'un « soft power » russe. Les troupes du MTchS sont ainsi intervenues sur la quasi-totalité des continents principalement suite à des tremblements de terre - comme en Iran en 1997 ou en Colombie en 1999 -, des inondations - comme en Corée du Nord en 2007 -, des incendies - comme en Grèce en 2000 - ou encore des catastrophes aériennes. Le MTchS constitue ainsi un instrument opportun de présence de la Russie à l'étranger, dans sa sphère classique d'influence, mais aussi au-delà.

Outre les interventions de sauvetage menées par les troupes du MTchS à l'étranger, la promotion du savoir-faire russe en matière de protection civile passe par la création de structures jumelles du MTchS à l'international, selon un véritable mécanisme de transfert institutionnel (Bertrand 2009 : 95-104). Si des structures ont été créées avec le soutien de représentants du MTchS de Russie, principalement dans la CEI (Azerbaïdjan, Ossétie du Sud et Abkhazie), l'idée de créer de telles structures en Amérique Latine, au Venezuela par exemple, est évoquée. La politique humanitaire d'État institutionnalisée en Russie dès la première moitié de la décennie 1990 se présente comme le vecteur d'une politique d'influence, voire de puissance à l'intérieur de la Fédération, comme à l'international, dans un environnement où la figure du sauveteur supplanterait presque celle du soldat.

\section{Bibliographie}

Andreev 2002 : Valentin Andreev, «Bjudžet MčS kak byl, tak i ostalsja tajnoj », Kompromat, 10.11.2002. Article disponible à l'adresse suivante : http://www.compromat.ru/ page_12445.htm (dernière consultation : octobre 2013)

Beck 2008 : Ulrich Beck, La société du risque : sur la voie d'une autre modernité, Paris : Flammarion, 2008.

Bertrand 2009 : Eva Bertrand, Humanitaire ou sécuritaire? La politique humanitaire d'État en Russie postsoviétique : étude de l'institutionnalisation du ministère des Situations d'Urgence, Mémoire de master dirigé par Françoise Daucé et Jean-Robert Raviot, IEP de Paris, 2009.

Brauman 1995 : Rony Brauman, L'action humanitaire, Paris : Flammarion, coll. Dominos, 1995.

Dubois 1999 : Vincent Dubois, La politique culturelle : genèse d'une catégorie d'intervention publique, Paris : Belin, 1999.

Dubuet 2003 : Fabien Dubuet, « Le mythe de l'humanitaire d'État », Revue humanitaire, Printemps/Eté 2003, n 7 , pp. 53-59.

Elie 2013 : Marc Elie, « Late Soviet Responses to Disasters, 1989-1991 : A New Approach to Crisis Management or the Acme of Soviet Technocratic Thinking ? ", The Soviet and Post-Soviet Review, Vol. 40, Issue 2, 2013, pp. 214-38.

Favarel-Garrigues et Startsev 2007 : Gilles Favarel-Garrigues et Iaroslav Startsev, « La combinaison des héritages et des emprunts dans la transformation de l'administration russe », Critique internationale, avril-juin 2007, $\mathrm{n}^{\circ} 35$, pp. 69-85.

FOM 2002 : FOM. « MčS obraz spasatelja », enquête d'opinion du Fond d'Études de l'Opinion publique du 26.09.2002, document en ligne consulté en octobre 2013 : http://bd.fom.ru/ report/map/az/0-9/495_1426/dd023825\#d023825.. 
Hassenteufel 2008 : Patrick Hassenteufel, Sociologie politique : l'action publique, Paris : Armand Colin, 2008.

Lascoumes 1999 : Pierre Lascoumes (dir.), Instituer L'environnement : vingt-cinq ans d'administration de l'environnement, Paris : L'Harmattan, 1999.

Lesage 1993 : Michel Lesage, "La réforme administrative graduelle en Russie », Revue française d'administration publique, octobre-décembre 1993, n 68, pp. 619-630.

Maev 2002 : Igor Maev, « Prizyv vesennij », Spasatel’ MčS Rossii, mars-avril 2002, n 3-4 (19-20), p. 16.

Mandrillon 1991 : Marie-Hélène Mandrillon, « Les voies du politique en URSS. L'exemple de l'écologie », Annales. Économies, Sociétés, Civilisations, 1991, vol. 46, nº 6, pp. 13751388 .

Merlin 2003 : Aude Merlin, « L'humanitaire d'État civil en Russie : urgence intérieure ou instrument de politique extérieure ", Revue humanitaire, Printemps/Eté 2003, n 7 , pp. 87-103.

Muhin 2013 : Vladimir Muhin, «Sergej Šojgu vozvrašaet sebe MčS », Nezavisimaja gazeta, 22.08.2013. Article en ligne consulté en octobre 2013 : http://www.ng.ru/ armies/2013-08-22/1_shoigu.html.

Porfiriev 1998 : Boris Porfiriev, Disaster Policy and Emergency Management in Russia, New York : Nova Science Publishers Inc., 1998.

Porfiriev et Simons 2012 : Boris Porfiriev, Greg Simons, Crises in Russia. Contemporary Management Policy and Practice from a Historical Perspective, Burlington : Ashgate, 2012

Renz 2007a : Bettina Renz, « Crisis Response in War and Peace : Russia's 'Emergencies' Ministry and Security Sector Reform », World Defence Systems, n 17, 2007, pp. 148152.

Renz 2007b : Bettina Renz, «The siloviki in Russian Politics : Political Strategy or a Product of the System ? », Russian Analytical Digest, 20 March 2007, n 17, p. 2-5.

Rohart 2005 : Ludovic Rohart, La représentation politique et professionnelle des sapeurspompiers de la Troisième République à nos jours, Thèse de doctorat sous la direction de Frédéric Sawicki, Université de Lille 2, 2005.

Ržeševskij 2005 : Aleksandr Ržeševskij, Živaja voda Eniseja. Sergej Šojgu : stranicy biografii, Moscou : Geroi Otečestva, 2005.

Choïgou 2000 : Sergueï Choïgou, « K-10 letiju MČS Rossii. Simvol nadežnosti i nadeždy », Graždanskaja zaščita, n 12,2000 , pp. 3-5.

Streltsina 2003 : Milanna Streltsina, « Rol' MTchS v sostave ispolnitel'noj vlasti Rossii », Političeskaja nauka, 2003, n 1 , pp. 145-164.

Streltsina 2006 : Milanna Streltsina, « Politics of Agency Design in Post-Soviet Russia : Case of Ministry for Extraordinary Situations », 15.03.2006. Disponible à l'adresse suivante : http://www.zdes.spb.ru/text/Streltsina_Politics\%20of\%20Agency\%20Design.pdf (dernière consultation : décembre 2008).

Taylor 2007 : Brian Taylor, Russia's Power Ministries : Coercion and Commerce, Syracuse University : Institute for National Security and Counterterrorism, October 2007.

Thomas 1995 : Timothy Thomas, « EMERCOM : Russia's Emergency Response Team », Low Intensity Conflict and Law Enforcement, Autumn 1995, vol. 4, n² 2, pp. 227-236. Article en ligne consulté en octobre $2013:<$ http://www.fas.org/nuke/guide/russia/ agency/rusert.htm>.

Tsyganok 2010 : Anatolij Tsyganok, « Požarnaja trevoga », Polit.ru, 19 septembre 2010. Article en ligne consulté en octobre $2013:<$ http://www.polit.ru/author/2010/08/04/pozary. html>. 


\section{Eva BERTRAND}

Volkov 2002 : Vadim Volkov, « Security and Enforcement as Private Business : the Conversion of Russia's Power Ministries and its Institutional Consequences », in Victoria Bonnell and Thomas Gold, The Entrepreneurs of Europe and Asia : Patterns of Business Development in Russia, New York : M.E Shape, 2002, pp. 83-103.

VTsIOM 2013 : VTsIOM, « Rejting raboty rossijskih ministrov », 17.10.2013. Document en ligne consulté en octobre $2013:<$ http://wciom.ru/index.php?id=459\&uid=114567>. 